\title{
Perfil epidemiológico de pacientes com catarata traumática no Hospital de Olhos do Paraná
}

\author{
Epidemiological aspects patients with traumatic cataract \\ who sought care in Hospital de Olhos do Paraná
}

Maria Celina Salazar Rubim Pereira ${ }^{1}$, Michele Aparecida Lonardoni Krieger ${ }^{2}$, Ana Cláudia Mariushi ${ }^{3}$, Hamilton Moreira ${ }^{4}$

\section{RESUMO}

Objetivo: Avaliar os resultados anatômicos e visuais de pacientes com catarata traumática atendidos no Hospital de Olhos do Paraná, apresentando ou não alterações oculares associadas e que foram submetidos ao tratamento cirúrgico, além de traçar o perfil analisando tipo de trauma, relação entre ocupação e trauma, relação entre tipo de trauma e prognóstico visual, correlacionando acuidade visual (AV) pré e pós-operatória. Métodos: Após minuciosa avaliação oftalmológica foram coletados os seguintes dados: sexo, idade, ocupação, tipo de trauma, AV pré e pós-tratamento, alterações oculares associadas e dos pacientes que foram submetidos à cirurgia as complicações pós-operatórias mais frequentes. Resultados: Foram atendidos 45 pacientes com catarata traumática. Destes, 25 submeteram-se à cirurgia. A maioria dos pacientes era do sexo masculino (88\%), sofreu trauma contuso (52\%) e apresentou idade média de 43,16 anos, atingindo uma faixa etária economicamente ativa. Estes traumas foram classificados como: laboral (14), recreacional, doméstico e automobilístico. Subluxação de cristalino foi a alteração ocular associada mais observada. Vinte e dois pacientes $(88 \%)$ apresentaram visão inicial $\geq 20 / 400$, entre os submetidos à cirurgia, 64\% apresentaram melhor acuidade visual corrigida pela tabela de Snellen (MAVC) $\leq 20 / 40$. Opacidade cápsula posterior foi a complicação pós-operatória mais frequente. Conclusão: O perfil dos pacientes atendidos, no HOP, com catarata traumática é, na grande maioria, homens com idade média de 43 anos, vítimas de trauma contuso ocorridos quando desenvolviam atividades laborais, expostos ao risco de acidente sem equipamentos de proteção, atingindo principalmente agricultores, pedreiros e serralheiros. Esses, após o tratamento cirúrgico apresentaram melhora significativa da visão.

Descritores: Catarata; Ferimentos e lesões; Traumatismos oculares/classificação; Traumatismos oculares/cirurgia; Subluxação cristalino/cirurgia; Resultado de tratamento

\section{ABSTRACT}

Objective: To evaluate the anatomic and the visual results of patients with traumatic cataract who sought care in Hospital de Olhos do Paraná (HOP), that were subjected to surgical treatment in this service. To describe their epidemiological and clinical aspects, and to analyze the post operative functional results. Methods: There were included in the study all patients subjected to a surgical treatment and the following characteristics were analyzed: sex, age, pre and postoperative visual acuity, mechanism of trauma, associated ocular alterations and most frequent postoperative complications. Results: Forty five patients were treated with clinical diagnosis of traumatic cataract during the study period. From the total, 25 were submitted the surgical treatment. The majority of patients were male (88\%), a history of blunt trauma could be ascertained (52\%) middle age $43 \pm 16$ years reaching an economically active group. Manual laborers were the most frequent (14), followed by recreational accidents, home accidents and automobilistic accidents. Traumatic subluxation lens were the most frequent ocular alterations associated. Twenty two patients $(88 \%)$ presented pre-operative visual acuity $\geq 20 / 400$. Among the patients that were submitted to cataract surgery, $64 \%$ showed better visual acuity corrected by table of Snellen $\leq 20 / 40$. Cause of poor post operative visual acuity posterior capsule opacity Conclusion: In this study, the majority of patients were male, with mean age of $43 \pm 16$ years and victims of blunt trauma during their job without equipments of protection, reaching principally farmers, masons and locksmiths. It was observed a clear improvement of postoperative visual acuity among these patients.

Keywords: Cataract; Wound and injuries; Eye injuries/classification; Eye injuries/surgery; Lens subluxation; Treatment outcome

\footnotetext{
${ }^{1}$ Residente do $3^{\circ}$ ano de Oftalmologia Do Hospital de Olhos Do Paraná - Curitiba (PR), Brasil;

${ }^{2}$ Residente do $1^{\circ}$ ano de Oftalmologia do Hospital de Olhos do Paraná - Curitiba (PR), Brasil;

${ }^{3}$ Hospital de Olhos do Paraná - Curitiba (PR), Brasil;

${ }^{4}$ Doutor Professor Adjunto da Universidade Federal do Paraná (UFPR) - Curitiba (PR) - Brasil; Professor Titular da Faculdade

Evangélica do Paraná (FEPAR) - Curitiba (PR), Brasil.

Trabalho realizado no Hospital de Olhos do Paraná - Curitiba (PR) - Brasil
}

Os autores declaram inexistir conflitos de interesse

Recebido para publicação em 20/5/2011 - Aceito para publicação em 19/10/2011 


\section{INTRODUÇÃO}

A catarata é uma das principais complicações das lesões traumáticas do globo ocular, sendo consequência de alterações da transparência do cristalino. A catarata traumática é a principal causa de cegueira unilateral em pessoas jovens e na faixa etária economicamente ativa ${ }^{(1-4)}$.

A incidência de catarata traumática na literatura varia de 30 a $65 \%$, estando a sua faixa etária de aparecimento, em média, duas décadas inferiores ao aparecimento da catarata senil. A prevalência dos traumas oculares é maior no sexo masculino, variando na literatura de 76 a 97\%, e esta variação depende do local de ocorrência e tipo de trauma ${ }^{(3,5)}$.

As cataratas traumáticas não são necessariamente oriundas de traumas contusos ou perfurantes. Outros fatores podem estar envolvidos na gênese deste quadro, tais como: choque elétrico, variação térmica, radiação ionizante, radiação infravermelho. Este tipo de catarata não se trata de uma patologia rara e, frequentemente, os olhos acometidos apresentam uma variedade de alterações morfológicas e funcionais dos segmentos anterior e posterior ${ }^{(1,3)}$.

O procedimento exigido pela catarata traumática apresenta-se como um desafio para os cirurgiões de segmento anterior em virtude da grande quantidade de elementos que se deve levar em consideração, merecendo do oftalmologista uma atenção especial para com a correta abordagem e escolha da conduta, seja esta clínica ou cirúrgica ${ }^{(2)}$.

Apesar de o planejamento ser fundamental, frequentemente a surpresa e a improvisação estão presentes na cirurgia da catarata traumática. A evolução dos recursos tecnológicos e das técnicas cirúrgicas vêm trazendo uma melhoria importante no seu prognóstico, proporcionando uma recuperação significativa da acuidade visual em muitos casos. Contudo, traumas severos ainda apresentam resultados reservados ${ }^{(4)}$.

O objetivo deste trabalho é avaliar os resultados anatômicos e visuais de pacientes com catarata traumática, que buscaram atendimento no Hospital de Olhos do Paraná (HOP), apresentando ou não alterações oculares associadas e que foram submetidos a tratamento cirúrgico neste serviço. Bem como traçar um perfil destes pacientes analisando tipo de trauma, relação entre ocupação e trauma, relação entre o tipo de trauma e prognóstico

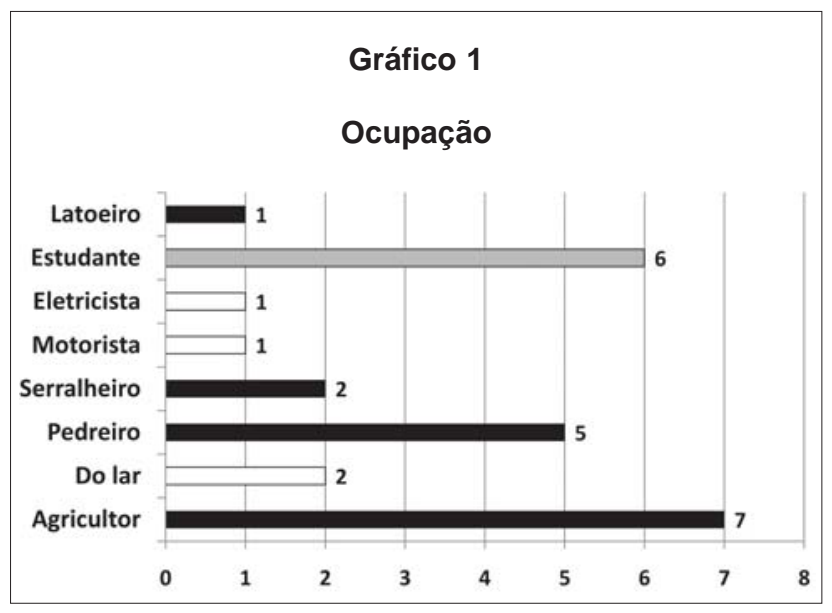

visual, o tempo decorrido entre o acidente e a cirurgia, correlacionando acuidade visual pré e pós-operatória.

\section{Métodos}

O estudo foi realizado no Hospital de Olhos do Paraná, na cidade de Curitiba, onde são atendidos em média de 12 mil consultas/mês, entre as consultas próprias e encaminhamento de outros serviços referenciados de oftalmologia da cidade e região. A pesquisa foi realizada no período de abril de 2009 a julho de 2010, período em que foram diagnosticados pacientes com catarata traumática.

Os pacientes foram submetidos a uma minuciosa avaliação oftalmológica na qual foram coletados os seguintes dados: sexo, idade, ocupação, tipo de trauma, acuidade visual (AV) prévia ao tratamento, alterações oculares associadas, tipo tratamento, tempo decorrido do trauma até o atendimento inicial, AV pós-tratamento e dos pacientes que foram submetidos ao tratamento cirúrgico as complicações pós-operatórias mais frequentes.

A análise dos resultados foi obtida com estatística descritiva, com uso de limites mínimos e máximos, porcentagem, mediana, se comparou a melhor acuidade visual corrigida (MAVC) dos pacientes operados de catarata traumática associada ou não a outras lesões oculares sem comparar a técnica cirúrgica empregada. A análise da MAVC pré e pós-operatórias foi realizada através do teste de Wilcoxon. Também foi analisada a relação entre tipo de trauma e ocupação dos pacientes através do teste qui-quadrado, e a relação do prognóstico visual com o tipo de trauma. A acuidade visual destes pacientes foi avaliada com melhor correção óptica e mensurada pela tabela de Snellen.

$\mathrm{O}$ critério usado para inclusão foi pacientes portadores de catarata traumática. E os critérios de exclusão foram pacientes que apresentassem aumento escavação de nervo óptico no olho acometido, melhora da acuidade visual com lente de contato, acuidade de 20/30 com melhor correção ou ainda que perdessem segmento do acompanhamento clínico.

\section{Resultados}

Foram atendidos 45 pacientes com diagnóstico clínico de catarata traumática durante o período do estudo. Deste total, 13 perderam seguimento clínico, sendo,

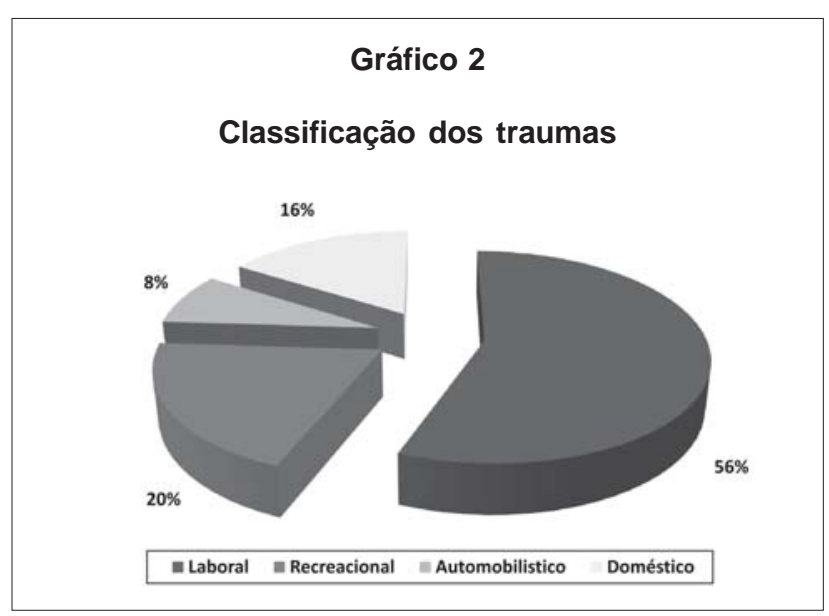




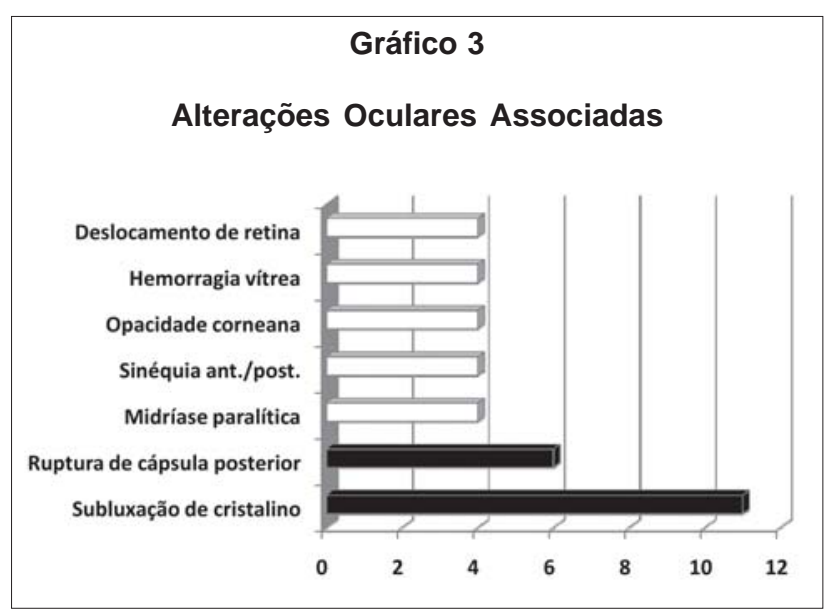

portanto, excluídos do estudo; 3 pacientes $(6,66 \%)$ obtiveram melhora da AV com o uso de lente de contato rígida; 1 paciente $(3,44 \%)$ teve contraindicação cirúrgica pelo aumento de escavação do nervo óptico do olho comprometido, 3 pacientes $(6,66 \%)$ tiveram cirurgia contraindicada devido ao descolamento total de retina. Dentre as 45 pessoas com diagnóstico de catarata traumática que buscaram atendimento no HOP, 25 (55,5\%) foram submetidos ao tratamento cirúrgico.

Entre os 25 pacientes submetidos ao tratamento cirúrgico, 22 (88\%) eram homens e apenas 3 (12\%) mulheres. A idade média destes pacientes foi de 43,16 anos de idade, atingindo uma faixa etária economicamente ativa, 7 $(28 \%)$ eram agricultores, $6(24 \%)$ estudantes e $5(20 \%)$ pedreiros (Gráfico 1). Trauma contuso foi o mais prevalente neste estudo compreendendo $52 \%$ dos tipos de trauma, seguido por trauma perfurante (44\%) e choque elétrico (4\%). Estes traumas foram classificados como: laboral (14), recreacional (5), doméstico (4) e automobilístico (2), (Gráfico 2). O tempo médio transcorrido entre o trauma e a busca por atendimento médico foi de 12,6 anos.

A subluxação de cristalino (44\%) apareceu como a alteração ocular associada à catarata traumática mais frequente, seguida por ruptura de cápsula posterior $(24 \%)$, midríase paralítica, sinéquia anterior/posterior, opacidade corneana, descolamento de retina e hemorragia vítrea com $16 \%$ (Gráfico 3 ).

Em relação ao procedimento cirúrgico realizado, 14 pacientes $(56 \%)$ foram submetidos à facoemulsificação com implante de lente intraocular, 5 pacientes $(20 \%)$ foram submetidos à facectomia extra-capsular com implante de lente intraocular, 1 paciente $(4 \%)$ foi submetido à facoemulsificação sem implante de lente intraocular e 2 pacientes $(8 \%)$ submetidos à facectomia intracapsular sem implante de lente intraocular. Houve ainda 3 pacientes $(12 \%)$ que foram submetidos à vitrectomia, destes apenas 1 foi submetido a implante secundário de lente intraocular e 2 permaneceram afácicos. As principais complicações pós-operatórias que ocorreram foram opacidade de cápsula posterior $(32 \%)$ e reação de câmara anterior (16\%) (Gráfico 4).

No período pré-operatório, 3 pacientes (12\%) apresentaram visão entre 20/60 e 20/200 e 22 pacientes (88\%) apresentaram visão menor ou igual a 20/400. No pósoperatório 16 pacientes (64\%) apresentaram visão mai-

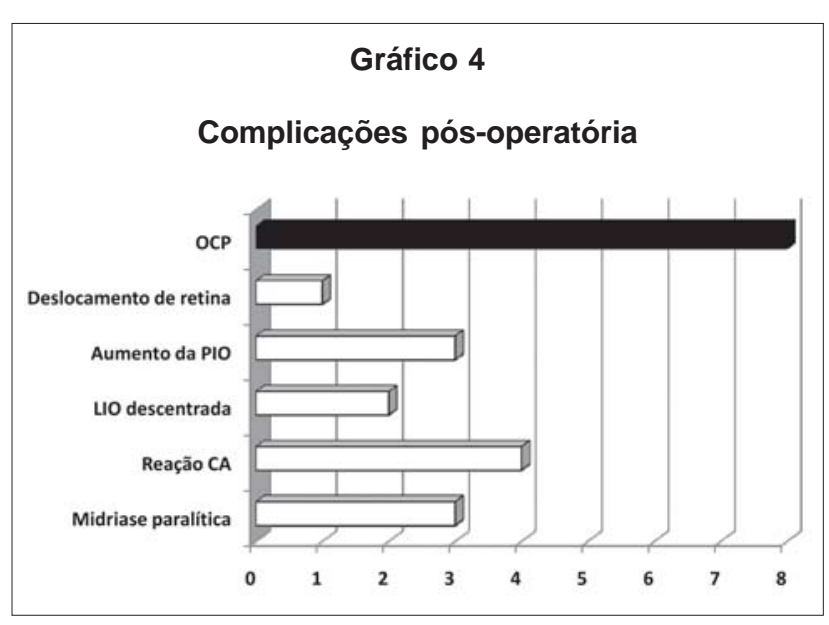

Tabela 1

Acuidade Visual

\begin{tabular}{ccc}
\hline & \multicolumn{2}{c}{ Número de olhos (\%) } \\
\hline MAVC & Pré-operatória & Pós-operatória \\
\hline$\geq 20 / 40$ & $0(0)$ & $16(64)$ \\
$20 / 60-20 / 200$ & $3(12)$ & $5(20)$ \\
$\leq 20 / 400$ & $22(88)$ & $4(16)$ \\
\hline
\end{tabular}

MAVC $=$ Melhor acuidade visual corrigida medida na tabela de Snellen

or ou igual a $20 / 40,5$ pacientes $(20 \%)$ apresentaram visão entre $20 / 60$ e 20/200 e 4 pacientes (16\%) apresentaram visão menor ou igual a 20/400 (Tabela 1).

\section{DisCusSÃO}

A incidência de 30 a $65 \%$ de catarata traumática, na literatura, varia baseado na seleção de pacientes e critérios de inclusão em cada estudo. Tanto nos traumas contusos como nos perfurantes, o tratamento cirúrgico deve ser bem direcionado, com a finalidade de se obter menor resposta inflamatória com a colocação da lente intraocular $(\mathrm{LIO})^{(2-5)}$.

Uma anamnese bem feita do tipo de trauma, e dos antecendentes do trauma permite determinar a dificuldade da cirurgia e planificar previamente a estratégia cirúrgica e o prognóstico visual ${ }^{(2,6-13)}$.

A decisão de tratar cirurgicamente a catarata deve ser individualizada no processo de conhecer o estado integral do globo ocular. A abordagem cirúrgica da catarata traumática quase nunca implica numa situação de emergência, logo pode ser manejada de forma eletiva( ${ }^{(8-10)}$.

Como em estudos prévios, observamos maior incidência do sexo masculino. Dos 25 pacientes que se submeteram à cirurgia, 22 pacientes $(88 \%)$ eram do sexo masculino e $3(12 \%)$ do sexo feminino. Na literatura são relatadas taxas de incidência no sexo masculino variando de $88,37 \%$ a $54,54 \%$ e no sexo feminino de $15,76 \%$ a $54,54 \%{ }^{(2,49,11)}$.

Cinquenta e seis por cento dos pacientes que 
procuraram o hospital de olhos tinham idade superior a 35 anos, com uma idade média de 43/16 anos de idade, atingindo na grande maioria dos casos uma faixa etária economicamente ativa, o que torna esse tipo de trauma um problema de saúde pública. Dos 25 pacientes, 14 sofreram o trauma, que causou catarata, enquanto trabalhavam, sendo classificados como trauma laboral. Essa classificação acomete, na maioria dos casos agricultores $(28 \%)$, seguidos por pedreiros e serralheiros. Há ainda um grupo, classificado como trauma recreacional (5), isto é, pessoas que sofreram o trauma ainda na infância, enquanto brincavam; o trauma doméstico (4) e o trauma automobilístico (2).

A literatura relata que os resultados cirúrgicos em adultos, vítimas de trauma durante a infância, sem anormalidades do segmento posterior e ambliopia, e submetidos à cirurgia mesmo muitos anos após o trauma, que neste referido estudo variou entre 28 a 81 anos após o trauma inicial, é relativamente segura e frequentemente resulta em uma melhora importante da acuidade visual ${ }^{(12,13-16)}$.

Nos acidentes de trabalho, a população mais acometida encontra-se na faixa etária entre 16 e 62 anos. A alta incidência dos casos de acidentes de trabalho (56\%) está relacionada ao baixo nível sócioeconômico da população estudada, por se tratar de um ambulatório público e à falta de uso de equipamentos de proteção individual (EPI) para segurança do trabalho. A baixa porcetagem dos acidentes automobilísticos $(8 \%)$ é um reflexo da obrigatoriedade do uso do cinto de segurança. A Associação Brasileira de Medicina de Tráfego, atualmente, afirma que houve $68 \%$ de redução dos traumas faciais com a obrigatoriedade do uso do cinto de segurança. Além disso, a utilização da air bag nos veículos mais modernos também colabora na prevenção de tais traumatismos ${ }^{(5,12,17,18)}$.

Quando avaliada a etiologia do trauma, o tipo contuso ocorreu em 13 pacientes $(52 \%)$, já o perfurante em $11(44 \%)$ e por choque elétrico em 1 paciente $(4 \%)$. Este resultado é compatível com a grande maioria dos estudos revisados que relatam uma incidência de $75 \%$ de traumas contusos, somente dois desses estudos apresentaram o trauma perfurante como mais prevalente $\mathrm{e}^{(3,5,8,10,19,20)}$. Neste estudo foi analisado a relação entre o tipo de trauma e a MAVC pós-operatória e não houve diferença significativa $(\mathrm{p}=0.236)$ para MAVC pós-operatória quando comparamos trauma contuso ou perfurante.

O tempo transcorrido entre o trauma ocular e a indicação cirúrgica foi muito variável, entre 5 horas a 61 anos, tendo tempo médio entre o trauma e a busca por atendimento médico de 12,6 anos. Reggi et al. obtiveram em seus estudos de 60 casos de catarata traumática uma mediana de 1 ano, a partir do trauma até o atendimento oftalmológico $^{(5)}$. Em um estudo realizado na Índia por Shah et al., foi observado que o precoce atendimento após trauma ocular é significativamente determinante para o prognóstico visual final ${ }^{(12)}$. Verificamos que no trabalho realizado no Hospital de Olhos do Paraná, os pacientes em sua maioria, não apresentavam conhecimento sobre os recursos oftalmológicos para beneficiar sua visão logo após o trauma ocular, corroborando com a demora pela procura do atendimento especializado.

Das alterações oculares associadas, a mais comum foi a subluxação de cristalino, num total de 44\%. Dos 11 pacientes com subluxação cristaliniana que procuraram o serviço foi constatada ao exame oftalmológico inicial uma acuidade visual inferior ou igual a 20/200, isto é: conta dedos (CD), movimento de mãos (MM), percepção luminosa (PL), sem percepção luminosa (SPL) e 20/ 400. Estes pacientes foram submetidos ao tratamento cirúrgico seja facectomia ou facoemulsificação, tendo $88 \%$ dos procedimentos citados a colocação de lente intraocular (LIO), e 12\% sem implante de LIO. No geral houve uma melhora importante da acuidade visual, $63 \%$ dos pacientes ficaram com uma MAVC maior ou igual a 20/40. Aragonés Cruz ${ }^{(7)}$ mostra em seu estudo que $37 \%$ dos pacientes apresentavam subluxação do cristalino, seguido de $18 \%$ com desinserção da cápsula posterior e $14 \%$ de leucoma corneano. O autor reforça a importância da procura de sinais indicativos da subluxação de cristalino, como facodonese e iridodonese, pois se o cristalino estiver luxado há uma grande chance da presença de vítreo em câmara anterior. Estes achados são fundamentais para que se possa ter um planejamento cirúrgico mais preciso como, por exemplo, avaliar a necessidade do uso de um anel endocapsular para casos de deficiência zonular progressiva e grave.

Em 1998, Cionni e Osher ${ }^{(15)}$ introduziram o uso do anel endocapsular modificado. Este incorporou ao anel endocapsular original um gancho que possibilita a fixação na esclera sem violar a integridade do saco capsular, podendo ser utilizado para mais de 120 graus de lesão zonular. Várias vantagens do anel endocapsular têm sido descritas na cirurgia de catarata traumática. Dentre elas, temos: estabilização da cápsula e aumento da segurança e eficácia durante a facoemulsificação e implante da LIO, manutenção do contorno circular do saco capsular, proporcionar um suporte adicional ao saco capsular, pois empurra o equador do cristalino para a posição original, o que ajuda a impedir a descentração e contração em casos de zônula frágil e fibrose capsular ${ }^{(4,14,15,21)}$.

O teste de Wilcoxon, utilizado para verificar a melhora da acuidade visual, apresentou um nível de significância de $\mathrm{p}<0.001$ para pacientes submetidos ao tratamento cirúrgico. Isto prova uma melhora significativa da acuidade visual pós-operatória. Oitenta e oito por cento dos pacientes procuraram o serviço do Hospital de Olhos com uma visão menor ou igual a 20/400, seguidos de $12 \%$ com visão entre $20 / 60$ e 20/200. Após o procedimento cirúrgico de retirada da catarata traumática, 64\% ficaram com uma visão maior ou igual a 20/40; $5(20 \%)$ pacientes ficaram com uma acuidade visual entre $20 / 60$ e 20/200, sendo que destes $60 \%$ evoluíram para opacidade de cápsula posterior como complicação pós-operatória, 20\% ficaram afácicos e 20\% evoluíram com descolamento de retina subtotal.

Diversos trabalhos na literatura apontam resultados visuais satisfatórios com implante de lente intraocular no tratamento da catarata traumática, independente do tempo e técnica cirúrgica. Nestes a incidência da MAVC no pré-operatório menor ou igual a 20/60 varia de $84 \%$ a $100 \%$ dos pacientes e a MAVC pós-operatória melhor ou igual a $20 / 40$ varia de $43 \%$ a $94 \%$ dos pacientes ${ }^{(4,5,8,9,22)}$. No presente estudo, realizado no Hospital de Olhos do Paraná, dos pacientes que tiveram o implante intraocular $(80 \%)$ tinham na avaliação pré-operatória uma acuidade visual de $20 / 80$ ou pior, um total de $80 \%$; e a MAVC pós-operatória de $20 / 40$ ou melhor, um total de $75 \%$. 


\section{Conclusão}

O perfil dos pacientes que buscaram atendimento no HOP, portadores de catarata traumática, é na grande maioria do sexo masculino, com uma idade média de 43 anos, vítimas de trauma contuso em sua maioria. Esse trauma ocorreu, na maioria dos casos, enquanto estes pacientes desenvolviam suas atividades laborais, expostos ao risco de acidente e sem equipamentos de proteção, envolvendo principalmente agricultores, pedreiros, serralheiros e latoeiros.

No estudo proposto, não houve diferença significativa para MAVC pós-operatória quando comparada o trauma contuso com o perfurante.

$\mathrm{Na}$ análise dos pacientes vítimas de trauma ocular, a alteração ocular associada mais prevalente à catarata traumática foi a subluxação do cristalino em $44 \%$ dos casos.

Em relação ao tempo transcorrido entre o trauma ocular e a busca por atendimento, teve-se como tempo médio de 12,6 anos.

A MAVC destes pacientes antes do tratamento cirúrgico era na sua totalidade pior ou igual a 20/60 e após cirurgia $64 \%$ apresentaram MAVC melhor ou igual 20/40.

A catarata traumática exige cuidados especiais na sua avaliação inicial para o planejamento do tratamento. A evolução dos recursos tecnológicos e das técnicas cirúrgicas vem trazendo uma melhoria importante no seu prognóstico, apesar de em traumas severos os resultados ainda serem reservados.

O fundamental é que a catarata traumática pode ser prevenida com medidas convencionais de proteção ocular, que devem ser amplamente disseminadas para a conscientização da população em geral.

\section{RefERÊNCIAS}

1. Freitas JAH, Cardoso LM. Trauma ocular. Rio de Janeiro: Revinter; 2004.

2. Villar Kuri J, Montenegro Tapia T, Martínez Franco C, Aveleyra Fierro R, Sáez-Espínola F, Villaseñor Díez J, et al. Resultados visuales y anatómicos en pacientes operados de catarata traumática. Microcir Ocular. 2003;3.

3. Blum M, Tetz MR, Greiner C, Voelcker HE. Treatment of traumatic cataracts. J Cataract Refract Surg. 1996;22(3):342-6.

4. Marques DMV, Marques FF, Cionni RJ, Osher RH, Freitas LL. Avaliação do uso do anel endocapsular modificado em casos de subluxação traumática do cristalino. Arq Bras Oftalmol. 2007;70(5):746-51.

5. Reggi JR, Dantas MCN, Dantas PEC, Borges MJH. Catarata traumática: estudo de 60 casos. Arq Bras Oftalmol. 1997;60(5):489-92.

6. Shah MA, Shah SM, Shah SB, Patel UA. Effect of interval between time of injury and timing of intervention on final visual outcome in cases of traumatic cataract. Eur J Ophthalmol. 2011 Mar 24. [Epub ahead of print].
7. Aragonés Cruz B. Resultados en la aplicácion del tratamiento quirúrgico de la catarata traumática. Rev Cuba Oftalmol. 2007;20(2).

8. Leal FAM, Tartarella MB. Catarata pediátrica pós-trauma. Arq Bras Oftalmol. 2004;67(2):227-30.

9. Bekibeli CO, Fasina O. Visual outcome of traumatic surgery cataract in Ibadan, Nigeria. Niger J Clin Pract. 2008;11(4):372-5.

10. Meirelles A, Soriano E. Catarata traumática: resultados visuais e complicações. Arq Bras Oftalmol. 2003;66(Supl. 4):4171.[Resumos de temas livres, painéis e casos clínicos a serem apresentados no XXXII Congresso Brasileiro de Oftalmologia; 2003].

11. Tabatabaei A, Kiarudi MY, Ghassemi F, Moghimi S, Mansouri M, Mirshahi A, Kheirkhah A. Evaluation of posterior lens capsule by $20-\mathrm{MHz}$ ultrasound probe in traumatic cataract. Am J Ophthalmol. 2011 Aug 20. [Epub ahead of print].

12. Shah M, Shah S, Shah S, Prasad V, Parikh A. Visual recovery and predictors of visual prognosis after managing traumatic cataracts in 555 patients. Indian J Ophthalmol. 2011;59(3):217-22.

13. Centurión V, Nicoli C, Villar Kuri J. El libro del cristalino de las Américas. São Paulo: Santos; 2007.

14. Nishi O, Nishi K, Menapace R. Capsule-bending ring for the prevention of capsular opacification: a preliminary report. Ophthalmic Surg Lasers. 1998;29(9):749-53.

15. Cionni RJ, Osher RH. Endocapsular ring approach to the subluxed cataracts lens. J Cataract Refract Surg. 1995;21(3):245-9. Comment in J Cataract Refract Surg. 2001;27(11):1710-1.

16. Scherer WJ, Hauber FA. Surgical outcomes in adult patients after repair of anterior segment trauma sustained during childhood. J Cataract Refract Surg. 2001;27(2):256-60. Comment in J Cataract Refract Surg. 2002;28(1):6.

17. Bison SHDF, Reggi JRA. Traumas oculares: nosologia de 1171 casos. Arq Bras Oftalmol. 1995;58(2):105-11.

18. Lima VF. O cinto de segurança: proteção à integridade física e a eficácia da lei. Rev ABRAMET. 2002.

19. Synder A, Kobieslka D, Omulecki W. [Intraocular lens implantation in traumatic cataract]. Klin Oczana. 1999;101(5):343-6. Polish.

20. Rozsíval P, Hakenová J. [Results of 52 operations for traumatic cataracts]. Cesk Oftalmol.1992;48(5):325-30. Czech

21. Chee SP, Jap A. Management of traumatic severely subluxated cataracts. Am J Ophthalmol. 2011;151(5):866-871.e1.

22. Rumelt S, Rehany U. The influence of surgery and intraocular lens implantation timing on visual outcome in traumatic cataract. Graefes Arch Clin Exp Ophthalmol. 2010;248(9):1293-7.

\section{Autor correspondente:}

Hamilton Moreira

Rua Coronel Dulcídeo, $\mathrm{n}^{\circ} 199$ - 4Andar

CEP 80420-170 - Curitiba/PR, Brasil

Tel: (41)3310-4224/Fax: (41) 3310-4228

Email: hamiltonmoreira@mac.com;

mcrubim@hotmail.com; mi.lonardoni@hotmail.com 